\title{
MERCADO DE TRABAJO JUVENIL Y POLITICAS DE EMPLEO
}

\author{
Enric Sanchis \\ Universitat de València
}

\section{Resumen}

El paro juvenil es una de las caracteristicas distintivas de la nueva situacion que conocen muchas economias industrializadas desde 1973. En este artículo se reflexiona sobre las causas y consecuencias de la peor situacion que soporta el colectivo juvenil en el mercado de trabajo. Las causas bay que buscarlas más en las condiciones generales de la economia y del mercado de trabajo que en las caracteristicas individuales de los jóvenes. Las consecuencias repercuten sobre el concepto mismo de juventud y sobre los procesos de inserción de las nuevas generaciones en la sociedad. Finalmente, se reflexiona también sobre la filosofia que sustenta la política de empleo juvenil.

Resum

L'atur juvenil és una de les caracteristiques distintives de la nova situacio que coneixen moltes economies industrialitzades des de 1973. En aquest article es reflexiona sobre les causes i conseqüiencies de la pitjor situació gue suporta el col. lectiu jutvenil en el mercat de treball. Les causes, cal buscar-les més en les condicions generals de l'economia $i$ del mercat de treball que en les caracteristiques individuals dels joves. Les conseqüencies repercuteixen sobre el concepte de joventut $i$ sobre els processos d'inserció de les noves generacions en la societat. Finalment, es reflexiona també sobre la filosofia que sustenta la politica d'ocupació juvenil.

Summary

The youth unemployment rate is one of the most distinctive characteristics of the new situation that most industrialized economies bave known since 1973. In the present article we will discuss the causes and consequences of the worst situation the youth is enduring on the work market. The causes are to be found in the general economc situation as well as in the work market rather than in the individual characteristics of the young. The consequences influence the concept of youth itself as well as the idea of integration of this youth into society. We will also discuss the philosophy on which the youth politics are based. 


\section{JUVENTUD Y TRABAJO}

El concepto de juventud es impreciso. Hace referencia a un colectivo que se define más fácilmente por lo que no es que por lo que es ${ }^{1}$. Se habla de jóvenes con respecto a aquellos individuos que han dejado de ser niños pero que todavía no son adultos, que ya no viven en la situación de dependencia absoluta típica de la infancia, pero que todavía no han alcanzado la situación de independencia propia de la madurez o de la vida adulta. Los jóvenes pueden definirse, por tanto, como aquellas personas que, no siendo niños, no tienen rodavía la auronomía personal que les permite constituir unidades familiares con independencia de sus familias de origen. La autonomía personal es una realidad pluridimensional que implica la asunción de responsabilidades en diversos dominios de la vida social. Ahora bien, estas responsabilidades no suelen adquirirse todas a un tiempo, ni tampoco son adquiridas necesariamente por todos los sujetos a la misma edad. Esta imprecisión hace que algunos niños abandonen el status de la infancia antes que la mayoría de sus coetáneos y que algunos "jóvenes" lo sigan siendo cuando la mayoría de sus coetáneos son ya adultos. En consecuencia, la juventud es, más que una situación social precisa, un proceso de tránsito entre dos posiciones sociales bien definidas: el status de niño y el status de adulto.

Si definimos a los jóvenes como el conjunto de individuos integrados en un determinado tramo de edad, a continuación habrá que matizar que ni están todos los que son ni son todos los que están. Si estudiamos las características distintivas del colecrivo juvenil con respecto al colectivo adulto, enseguida habrá que reconocer que las diferencias no son tantas como las que podemos encontrar dentro de cada uno de esros colectivos, y que algunos grupos de jóvenes y adultos están caracteriológicamente más próximos entre sí de lo que pueden estarlo a otros grupos de sus respectivos colectivos. Los jóvenes no son una clase social. Hay jóvenes y jóvenes. Y esto es particularmente cierto cuando se esrudia a los jóvenes desde la perspecriva del mercado de rrabajo.

¿Dónde empieza y acaba la juventud? Desde el punto de vista del análisis laboral, el límite inferior viene dado de manera inequívoca por la legislación: se comienza a ser joven cuando se alcanza la edad mínima legal para trabajar. En estos momentos se es joven desde los 16 años, pero hace muy poco tiempo se era joven ya a los 14 años. Este límite operativo permite diferenciar netamente entre jóvenes y niños, pero no debe hacer olvidar que el trabajo no es una realidad ajena a la infancia. Hay niños ocupados en el trabajo negro de la economía sumergida, en el trabajo gris de las unidades productivas de carácter familiar $y$-fuera ya del dominio estricto de la producción- en el tra-

1. Galland, O. Les jeunes, La Decouverte, París, 1984. 
bajo fantasma de las tareas domésticas, a través de las cuales muchos (es mejor decir, muchas) abandonan el status de la infancia.

Por lo que se refiere al límite superior - que marca el final de la juventud y el comienzo de la vida adulta- el problema es más complejo. Hace veinte años un grupo de expertos habría definido a los jóvenes como el colectivo entre 14 y 19 años, no sin advertir que algunas tendencias apuntaban hacia la "juvenilizacion" del colectivo de 20 a 24 años. Hoy esos mismos expertos acabarían definiendo a los jóvenes como el colectivo de 16 a 24 años tras un encendido debate sobre si los de 25 a 29 años son o no también jóvenes. El concepto de juventud aquí utilizado excluye el grupo de 25 a 29 años por las siguientes razones: 1) Las tasas de paro, actividad y escolarización experimentan una fractura importante al cruzar la barrera de los 25 años, se asemejan más a las de los grupos adultos que a las del grupo de 20 a 24 años. Concretamente, la tasa de actividad se incrementa de manera notable mientras que las tasas de paro y escolarización se reducen de forma drástica. 2) La normativa laboral específicamente dirigida a los jóvenes, los define igualmente utilizando los 25 años de edad como límite superior. Por lo tanto, a nuestros efectos, son jóvenes las personas que tienen entre 16 y 24 años de edad.

$\mathrm{El}$ interés particular y creciente por la problemática laboral de los jóvenes es consecuencia directa de la crisis económica que estalla en 1973. Es a partir de esta fecha cuando empiezan a proliferar los estudios específicos sobre la materia y cuando se populariza el término «mercado de trabajo juveniln. Una característica distintiva de la segunda gran crisis económica de este siglo es que - -en relación con el empleo y el paro- ha incidido de forma particularmente dura sobre los jóvenes. Por el contrario, la crisis de 1929 afecta sobre todo a los obreros industriales, y sus efecros negativos sobre el empleo se repartieron de manera más homogénea entre los diferentes grupos de edad. Durante las tres décadas de fuerte crecimiento que experimentaron las economías industrializadas a partir del final de la segunda guerra mundial, la inserción de los jóvenes en la actividad económica tampoco planteó particulares dificultades ${ }^{2}$. Había dos vías básicas de integración laboral, una que conducía al mercado de trabajo obrero y otra que conducía al mercado de trabajo no obrero. Al mercado de trabajo obrero se accedía una vez acabada la escolarización obligatoria y tras empezar un período de aprendizaje que normalmente se realizaba in situ. Este período de aprendizaje se caracterizaba - como hoy en díapor la precariedad, no estaba a salvo de los abusos y, en España, era muy frecuente que se desarrollara al margen de la legislación laboral. Pero era relativamente breve $y$, tras una temporada de tanteos, incluso de varios cambios de

2. Roberts, K. School Leavers and their Prospects. Youth and the Labour Market in the 1980's, Open University Press, Milon Keynes, 1984. 
puesto de trabajo, parece que permitía al joven estabilizarse en un empleo con ciertas perspectivas económicas y profesionales; al menos con la perspectiva de alcanzar una posición social mejor que la de la familia de origen.

El mercado de trabajo no obrero estaba reservado para los jóvenes cuyas familias podían permitirse mantenerlos en el sistema educativo una vez superada la etapa obligatoria, desde los bachilleres hasta los universitarios. En consonancia con la expansión de la escolarización - que dará lugar finalmente a la escuela de masas- esta vía resulta cada vez más frecuentada y permite a los jóvenes acceder a una cuota de empleos también en expansión y, en principio, más satisfactorios. La expansión del mercado de trabajo no obrero está directamente relacionada con el crecimiento del sector público (particularmente educación y sanidad) y - más en general- con la modernización y burocratización de las unidades productivas. Dicho en otras palabras, con el cambio tecnológico (que incorpora un número creciente de cuadros técnicos al proceso productivo) y con la terciarización de la economía, tanto desde el punto de vista de los sectores productivos como desde el punto de vista de las profesiones.

Cuando se alcanza el punto álgido de los «treinta años gloriosos de crecimienton incluso algunos itinerarios obreros cualificados se inician fuera de las unidades productivas, en el sistema educativo. Son los años en que educación y empleo mantienen una relación casi idílica, que augura un matrimonio duradero. Poco tiempo después, la crisis económica se encargará de revelar que aquella sincronía fue excepcional y estaba condenada al divorcio.

La crisis económica significa la desaparición de millones de empleos en todo el mundo industrializado y el bloqueo de las dos vías de acceso al trabajo para las nuevas generaciones. Este hecho coincide con el asentamiento de la escuela de masas en las sociedades avanzadas: virrualmente toda la población en la edad correspondiente está encuadrada en la escuela obligatoria, pasa al menos diez años de su vida en el sistema educarivo, y al menos la mitad contintsa estudiando durante varios años una vez superado el nivel obligatorio. Están pues servidos todos los elementos para que comience a cuestionarse el funcionamiento del sistema educativo. El proceso semiauromático de absorción de las nttevas generaciones (ahora egresadas del sistema educativo) por parte del mercado de trabajo, se alarga y dramatiza, el período inicial de tanteos ya no está claro que conduzca a alguna parte. El paro juvenil se multiplica. Entre otras iniciativas de distinto origen, mediada la década de los setenta la Comunidad Europea pone en marcha los programas de transición a la vida activa. Ha nacido el mercado de trabajo juvenil ${ }^{3}$.

3. Planas I a đa inserció professional i social dels joves. Introducció als programes Transició de la CEEm, en Papers, 25, 1985. 
Puede hablarse de la existencia de un mercado de trabajo juvenil dentro del mercado de trabajo en su conjunto en la medida en que el paro y el empleo precario se encuentran aquí mucho más acentuados. Según los países, la tasa de paro juvenil duplica o triplica a la adulta con facilidad, y el porcentaje de jóvenes que trabaja en condiciones equiparables a las de los adultos se reduce: a valores cada vez más bajos. El método más fructífero de acercarse a la problemática del trabajo juvenil es la teoría de las segmentaciones. La crisis eco. nómica abre un período de incertidumbre que fuerza a las unidades produc. tivas a flexibilizar sus estructuras, incluido el uso del factor trabajo. La demanda de trabajo se articula en dos segmentos, uno estable y otro inestable, que movilizan respectivamente a los sectores fuertes y débiles de la fuerza de trabajo. Entre los primeros se encuentra la mayoría de los hombres adultos; entre los segundos, un porcentaje abrumador de jóvenes de ambos sexos.

Desde la perspectiva de la demanda, hay razones muy sólidas que permiten aceptar la existencia de fuerzas que tienden a consolidar un mercado de trabajo juvenil caracterizado por la precariedad. Piénsese, por poner sólo un ejemplo, en el distinto grado de incertidumbre que soportan las unidades productivas según trabajen en régimen de competencia o de oligopolio; según utilicen tecnologías avanzadas intensivas en capital o tradicionales intensivas en trabajo; o, dentro incluso de una misma unidad productiva, en las diferentes posibilidades de gestión de la mano de obra en función de la fase del proceso productivo a que se aplique. Sin embargo, desde la perspectiva de la oferta, la cuestión de si existe o no un mercado de trabajo juvenil —un lugar de paro y ocupaciones precarias sin perspectivas al que se ve abocada la mayoría de los jóvenes- es más discutible. El problema es el sistema de relaciones que se establece entre la precariedad laboral y los diferentes tipos de jóvenes; hasta qué punto la precariedad va a estar controlada por los jóvenes o va a ser una experiencia que marcará definitivamente el resto de su vida activa. Esta cuestión es fundamental y volveré sobre ella más adelante.

En estos momentos, no puede afirmarse con propiedad que estemos todavía en época de crisis. Todos los indicadores económicos señalan que nos encontramos de nuevo en una fase de crecimiento. Sólo los indicadores del mercado de trabajo persisten en recordar que algo no acaba de funcionar en la economía. Estamos en la "postcrisis", una fase caracterizada, a efectos de mercado de trabajo, por el hecho de que la secuencia beneficios/inversión/crecimiento económico/generación de empleo es insuficiente para reducir el paro a su llamada "tasa natural".

Además, la casi totalidad de los empleos creados no tiene nada que ver con lo que hasta hace muy pocos años se entendía que era un uempleon; desde luego no sirven para mantener una familia. Desde la perspectiva del mercado de trabajo juvenil, la época de postcrisis significa sobre todo un fuerte incremento 
de la tasa de rotación laboral de los jóvenes, la conversión de una parte importante del paro de inserción (el de los que buscan su primer empleo) en paro de movilidad ${ }^{4}$, y el incremento de la tasa de ocupación. Pero el paro continúa siendo un problema de primer orden. Tanto es así que la variable "sexo" ha dejado de ser el discriminador por excelencia en el mercado de trabajo, ese lugar es ocupado desde hace varios años por la variable "edad"s. La legislación específica sobre empleo juvenil ha venido a reforzar todos estos procesos.

\section{LAS EXPLICACIONES DEL PARO YUVENIL}

El problema del paro masivo es en gran medida un problema de paro juvenil, pero éste no registra la misma intensidad en todos los páses. No sólo es cierto que en algunos páses hay más paro que en otros, también lo es que en algunos países el paro es en mayor medida que en otros paro juvenil ${ }^{6}$. Este hecho ha estimulado el análisis de las causas específicas del paro juvenil. Se apuntan muchas de diverso significado ${ }^{7}$. En primer lugar, la estructura demográfica de la población, la circunstancia de que haya, relativamente, más jóvenes intentando entrar en el mercado de trabajo y menos adultos en edades próximas a la jubilación. El que unos países dispongan de una cuota de inmigrantes suficiente para cubrir el segmento precario del mercado de trabajo mientras que otros deben hacerlo recurriendo a algunos sectores de la población autóctona, en particular a los jóvenes. La consolidación en muchas unidades productivas de mercados de trabajo internos (a la misma empresa $o$ al sector) que cierran el acceso a quienes buscan su primer empleo o sólo les dejan escoger las ocupaciones devaluadas, que se concentran en los escalones más bajos de los distintos itinerarios laborales. Las prácticas discriminatorias de las empresas, que preferirian contratar a otro tipo de trabajadores antes que vérselas con jóvenes. La justificación económica de estas prácticas sería la menor productividad o el mayor coste de los trabajadores jóvenes con respecto a los adultos, un fenómeno que la evidencia empírica sigue sin poder confirmar o rebatir de manera concluyente ${ }^{8}$. Se habla igual-

4. Rose, J. En quête d'emploi, Economica, París, 1984.

5. Montoro, R. La inserción en la actividad econbmica: empleo y paro juvenil, Ministerio de Cultura, 1985.

6. Therborn, G. Por qué en algunos paises hay mas paro que en otros, Ediciones Alfons el Magnànim, IVEI, Valencia, 1989.

7. OCDE, El paro juvenil. Carsas y consecuencias, Ministerio de Trabajo, 1984.

8. Brown, P. y Ashton, D.N. (eds.), Education, Unemployment and Labour Markets, The Falmer Press, Londres, 1987; Junankar, P.N. (ed.), From School to Unemployment? The Labour Market for Young People, Macmillan Press, Londres, 1987; Marsden, D. y Ryan, P., "Where 
mente del distinto grado de protección sindical de que gozan jóvenes y adultos en el mercado de trabajo, porque el sindicato sirve sobre todo para defender a quien ya tiene un trabajo, no para procurarle uno a quien lo busca, porque el «último que entra es el primero que sale", porque las tasas de afiliación sindical alcanzan valores mínimos entre los jóvenes, etc. No se olvida tampoco citar la falta de formación de los jóvenes, pero sí se omite que tienen muchos más años de escolaridad que la mayoría de los adultos hoy en activo ${ }^{9}$. Se apunta también la falta de experiencia, sin explicar por qué esta circunstancia (que por definición acompaña a quien busca su primer empleo) no impidió la integración laboral de los jóvenes durante el período anterior a la crisis; y sin explicar igualmente cómo puede adquirirse experiencia si no es uexperimentando", es decir trabajando. Finalmente, se sugiere la posibilidad de que sean los nuevos valores y actitudes de los jóvenes ante el trabajo, unos y otros contrarios a la ética y a la disciplina laborales, los causantes de su mala posición en el mercado; argumento que olvida el hecho de que las encuestas registran porcentajes minimos de jóvenes refractarios al trabajo y, sobre todo, el hecho de que la mayoría de los jóvenes que trabajan lo hacen en las condiciones más duras ${ }^{10}$.

Muchos de los estudios sobre las causas específicas del paro juvenil experimenaan a lo largo de su desarrollo un giro muy curioso: comienzan preguntándose por qué los jóvenes son las víctimas principales de la situación del mercado de trabajo y acaban respondiéndose que las víctimas son los culpables. Si hay tanto paro juvenil es porque hay muchos (demasiados) jóvenes, o porque no están preparados para desempeñar los empleos que se les ofrecen $o$, simplemente, porque nada de lo que se les ofrece les parece lo suficientemente bueno para ellos. Este tipo de razonamiento debe evitarse de manera radical y no debe olvidarse que la "causa determinante" del paro juvenil es la misma que la del paro en general, a saber, que no bay trabajo para todos ' ${ }^{\prime}$. Dicho esro, cabe reconocer que debe existir algún tipo de "causas coadyuvantes" que expliquen por qué el paro se ceba particularmente con los jóvenes. Ahora bien, mi impresión es que la auténtica causa especifica del paro juvenil es la existencia (tácita) de un gran acuerdo social a todos los niveles para descargar sobre

do Young Workers Work? Youth Employment by Industry in Various European Economies", en British Journal of Industrial Relations, vol. 24, 1, 1986; Toharia, L. y Muro, J. "Es elevado el salario de los jóvenes?", en Economia y Sociologia del trabajo, 1/2, 1988.

9. Windolf, $P$. "L'expansion de l'enseignement et la surqualification sur le marché du travail", en Archives européennes de Sociologie, 1, 1984.

10. Sanchis, F. "Valores y actitudes de los jóvenes ante el trabajon, en Revista Española de Investigaciones Sociologicas, 41, 1988.

11. Touraine, A. y otros, ¿Qué empleo para los jövenes? Hacia estrategias innovadoras, Tecnos-Unesco, Madrid, 1988. 
"Papers": Revista de Sociologia

las generaciones jóvenes la mayor parte del coste social tanto de la crisis como de la actual reactivación económica.

El sistema de valores sobre el que se basa esta especie de macroacuerdo social no está falto de lógica ni de coherencia, tiene su legitimidad y no resulta fácil (ni quizás oportuno) cuestionarlo. Pero el sentido que pueda tener el consenso social en torno a la existencia de un mercado de trabajo juvenil comienza a desvanecerse cuando ese mercado deja de ser un episodio temporal en el itinerario laboral y vital de un individuo y se convierte en un corredor sin rerorno para un porcentaje creciente de jóvenes. Las posibles virtudes socializadoras que pueda tener la práctica de comenzar la vida laboral y adulta en condiciones adversas pierden su razón de ser cuando al final del camino no espera la recompensa de una posición laboral y social estabíe. En estas circunstancias, el mercado de trabajo juvenil puede acabar siendo una bomba de relojería colocada en los cimientos mismos de la sociedad.

\section{INTEGRACION LABORAL EINTEGRACIÓN SOCIAL DE LOS JOVENES}

El hecho de que los primeros contactos con el mundo del trabajo estén marcados por una experiencia de paro u ocupación precaria en empleos inestables, poco cualificados, infrapagados y sin perspectivas, constituye ya de por sí un grave problema. Pero hay más. Un sistema de relaciones industriales moderno no puede prescindir del pacto, del compromiso entre capital y trabajo. Ahora bien, con trabajadores resabiados por las primeras experiencias no es fácil entenderse, y sin entendimiento el objetivo de conseguir unidades productivas modernas, competitivas y ágiles para adaptarse a mercados cambiantes, queda seriamente en entredicho. $Y$ esto no es todo. El aspecto más preocupante de la inestabilidad laboral de los jóvenes son sus repercusiones sobre los procesos de integración social de las nuevas generaciones ${ }^{12}$.

La integración de los jóvenes en la sociedad ha seguido tradicionalmente dos grandes vías. La primera (masculina) ha estado siempre centrada en torno al llamado trabajo productivo; la integración social no se concebía al margen de la integración laboral. La segunda (femenina) ha tenido tradicionalmente como eje la familia, la maternidad y las labores domésticas. Otras vías, digamos alternativas o "psicodélicas", de integración social han sido siempre absolutamente minoritarias, incluso durante los juveniles y floridos años sesenta. Por razones que no vienen al caso, pero que no son exclusivamente de índole

12. Zárraga, J.L. Informe Juventud en España 1988, Instituto de la Juventud, Ministerio de Asuntos Sociales, 1989. 
cultural, la vía femenina ha entrado en franca decadencia, y durante los últimos años resulta cada vez menos frecuentada, años que coinciden casi puntualmente con la fase del paro masivo. En consecuencia, las dificultades laborales de los jóvenes se convierten automáticamente — hoy más que nunca-en dificultades de inserción social.

La integración en el mercado de trabaj̧o - desde la familia o desde el sistema educativo - nunca ha sido un camino de rosas y menos para todo el mundo. Pero antes la mayoría de los jóvenes conseguía superar las dificultades iniciales en un período relativamente breve. Sólo una minoría quedaba atrapada en la trampa de la precariedad y el desempleo. Y en muchos de estos casos el problema podía explicarse por factores externos al mercado de trabajo. La inestabilidad laboral era fruto de otras inestabilidades psicosociales de orden individual. Hoy esa minoría inestable ha pasado a convertirse en más de la mitad de los jóvenes activos. Pero la inestabilidad juvenil no es sólo de orden laboral. Si antes definíamos la juventud como un período transitorio entre dos status sociales seguros, estables y conocidos, ahora debemos reconocer que el status adulto es cada vez menos todo eso. La inestabilidad laboral no es ajena al universo adulto, muchos de cuyos habitantes saben que el empleo que ocupan ya no va a ser para toda la vida, al menos en su contenido. Se habla de la formación permanente, de la obsolescencia de los conocimientos anteriormente adquiridos y de la necesidad de estar preparados para el cambio, aunque la mayoría de los jóvenes sigue sin encontrar un trabajo digno de poner a prueba lo poco o mucho que pueden haber aprendido en el sistema educativo. Otras dimensiones de la vida social, como los modelos familiares o los referentes ideológicos, están igualmente en discusión, han dejado de ser pucrtos seguros hacia los que orientar la etapa de transición juvenil. Al final de la inestabilidad laboral y vital actual no se vislumbra más que una vida adulta marcada también por la inestabilidad ${ }^{13}$.

Quizás sea esta situación la que explica la forma particularmente dramácica como vive el paro y la precariedad la mayoría de los jóvenes. No es cuestión de comenzar a discutir qué tipo de paro (juvenil o no) tiene consecuencias más perversas sobre los individuos y la sociedad en su conjunto. Pero si debe recordarse que diversos estudios de psicología social han encontrado que el paro masivo actual ha tenido efectos tan demoledores sobre los individuos como los tuvo el que se produjo durante los años de la gran depresión ${ }^{14}$. Sin embargo, como se apuntaba más arriba, hay que distinguir entre diferentes tipos de jóvenes.

En función del grado de estructuración de los itinerarios educativos, del "capital" económico, social y cultural que puede poner en juego la famitia de

13. Garelli, F. La generazione della vita quotidiana, Il Mulino, Bolonia, 1984.

14. Jahoda, M. Empleo y desempleo. Un análisis sociopsicologico, Morata, Madrid, 1987 
origen, y de la disponibilidad o no de algunos referentes vitales sólidos, podemos diferenciar entre los jóvenes potencialmente en grado de mantener la inestabilidad laboral bajo control, y los jóvenes en peligro de ser engullidos definitivamente por esa inestabilidad laboral. El problema de la inestabilidad laboral de los jóvenes deviene particularmente grave cuando se combina con itinerarios vitales absolutamente desestructurados, es decir con la carencia de un proyecto de vida que dé algún sentido a la existencia. Inspirándonos en una investigación sobre la juventud catalana ${ }^{15}$, podriamos aventurar que la mitad de los jóvenes siguen itinerarios educativos y vitales orientados por una cierta lógica y por tanto gozan de cierra protección para hacer frente a las vicisitudes del mercado de trabajo; una cuarta parte está claramente instalada en posiciones de marginalidad laboral y social o en sus límites, y el resto se encuentra en una situación de indefinición que no permite pronosticar hacia cuál de los otros dos grupos va a decantarse en el futuro.

Una parte, incluso mayoritaria, de los jóvenes socialmente "protegidos" ante la precariedad laboral puede estar experimentando esta precariedad de manera "fisiológica" (se prueban diversas ocupaciones para poder elegir mejor o simplemente para disponer de dinero de bolsillo). Por el contrario, la mayoría de los jóvenes no protegidos van a sufrirla de manera claramente patológica: los primeros empleos no son el principio de nada, no les llevan a ninguna parre. Los candidatos a quedar atascados en posiciones marginales, tanto laborales como sociales, son los que atraviesan de manera insatisfactoria el nivel educativo obligatorio (en España el $30 \%$ de la población correspondien$\left.t^{16}\right)$, los hijos de los inmigrantes, de los parados ${ }^{17}$ y de los ocupados en profesiones obsoletas o en sectores en declive. Se trata de un colectivo de jóvenes, en aumento, con alto riesgo de exclusión social. La estrategia de intervención frente a ellos desborda el ámbito de la política laboral para entrar en el terreno educativo y en el de la política social.

\section{LAS POLITICAS DE EMPLEO JUVENIL}

La causa fundamental del paro juvenil es la misma que la del paro en su conjunto, a saber, que no hay trabajo para todos, que el nivel de demanda de trabajo es insuficiente para hacer frente a la oferta de trabajo. En este sentido la mejor terapia contra el paro juvenil es una acción decidida y voluntarista sobre el nivel

15. Casal, J. y otros, La inserción profesional y social de los jónenes. Los itinerarios de transición entre los 14 y los 25 años, ICE, Universidad Autónoma de Barcelona, 1989.

16. Díaz Malledo, J. La educación y el mercado de trabajo, Instituto de Estudios Económicos, Madrid, 1987.

17. Cabrer, B. Origen Social y paro juvenil, IVEI, Valencia, 1989, mccanografiado. 
de empleo: crear más empleo. En la medida en que la acción sobre la demanda de trabajo sea insuficiente, como de hecho ocurre, esta política debe ser acompañada por otra que actúe por el lado de la oferta con el objetivo de reducirla. Así pues, incrementar la demanda de trabajo, reducir la oferta $y$, además, estimular una mejor distribución del empleo existente (reduccion de la jornada laboral, potenciar las salidas temporales de actividad por motivo de formación, etc.).

Ahora bien, la situación del mercado de trabajo juvenil pone de maniffesto que los puentes tradicionales que conectaban familia y sistema educativo con el mundo del trabajo han sido dinamitados. No puede caerse en la ingenuidad de pensar que esos puentes van a levantarse de nuevo por sí solos una vez surta sus efectos la política general de empleo. Por otra parte está el problema del tiempo, qué hacer con los jóvenes en dificultades mientras vuelve -si es que lo hace algun día- la época del pleno empleo. Es por ello por lo que, desde mediados de los años setenta, en la OCDE comienzan a estudiarse estrategias específicas para el empleo juvenil. Y de hecho, diversos páses ponen en marcha dispositivos encaminados a facilitar la inserción de los jóvenes en la vida activa ${ }^{18}$.

La filosofía que subyace a todas las medidas adoptadas en el dominio de la política de empleo juvenil es doble: reducir el coste de la mano de obra juvenil e incrementar su nivel de formación.

Se supone que las dificultades específicas de los jóvenes en el mercado de trabajo derivan en gran medida de su coste o productividad con respecto al trabajo adul to y de sus carencias en cuanto a formación. Por lo que se refiere a la primera cuestión, ya hemos apuntado que hay evidencia empírica para todos los gustos; por tanto no hay evidencia empirica concluyente. Ocurre lo mismo que cuando se estudian las productividades comparadas de hombres y mujeres: las diferencias salariales denotan más prejuicios sociales que otra cosa. Y en mercados de trabajo como el español - que en este sentido no es ni mucho menos excepcional--, caracterizados por una cuota importantísima de empleos en los que la fuerza de trabajo alcanza la productividad óptima tras un breve periodo de rodaje, este tipo de argumentos no parecen demasiado sólidos.

En todo caso, el debate sobre el coste relativo del trabajo juvenil quizás tenía sentido - dentro y fuera de España - hace diez o quince años. Ahora no lo tiene en absoluto, dado que las unidades productivas disponen de amplias posibilidades para introducir mecanismos de discriminación sałarial en función de la edad. Supongamos que, efectivamente, entonces el trabajo juvenil fuese relativamente caro y que las correspondientes medidas que se han adoprado hayan sido justificadas. La misma OCDE se mostró muy cauta en cuanto a la conveniencia y posibles consecuencias de adoptar estas medidas. Las valoraba como

18. Hollister, R.G. y Freedman, D.H. "Programas especiales de empleo en ciertos países de la OCDE", en Revista Internacional de Trabajo, vol. 107, 3, 1988. 
moderadamente positivas siempre y cuando se limitasen a un pexíodo temporal relativamente breve. En caso contrario — se temía - podrían acabar desestabilizando socialmente a una generación (vinculado al tema del coste está el de la flexibilidad) y desplazando el epicentro del problema desde los jóvenes a otros colecrivos, como las mujeres adultas o los trabajadores de edad avanzada.

Algo de esto puede haber ocurrido ya efectivamente. Por tanto, quizás es hora de plantearse si no hay que empezar a desmantelar al menos algunos de estos dispositivos. La normativa laboral que permite la discriminación salarial en razón de la edad ha consagrado una nueva segmentación del mercado de trabajo que está reniendo repercusiones sobre la cohesión de la sociedad en el plano de las diferentes generaciones que la conforman y que está minando la base de legitimación de los sindicatos como interlocutores válidos del conjunto de la población trabajadora. Es utópico pensar que derogando la norma va a suprimirse automáticamente una segmentación que es anterior a ella y cuyas razones profundas van más allá de las disposiciones legales. Pero si no es realista plantearse a corto plazo la reunificación del mercado de trabajo ${ }^{19}$, tampoco lo es pensar que las actuales tendencias en curso pueden seguir prolongándose ad infinitum. ¿Qué tasa de precarización de la población ocupada, qué grado de debilitamiento de las instancias sindicales, puede permitirse un sistema de relaciones industriales que no puede prescindir de la implicación efectiva de los trabajadores para hacer frente al reto de la internacionalización de la economía y de la competirividad de las unidades producrivas? Hay que trabajar en la perspectiva de una deseable superación de la segmentación. Esto significa, hoy por hoy, que los interlocutores sociales han de constituir acuerdos sobre los grados de segmentación y los niveles de precarización tolerables, y que los dispositivos pactados para el empleo juvenil han de estar sometidos a control sindical. En la elaboración de esos acuerdos deberían participar los jóvenes, al menos desde la parte sindical. Las estructuras sindicales continúan reflejando en gran medida un mundo del trabajo de obreros industriales hombres y adultos que ya no corresponde a la realidad. La creciente terciarización de la economía significa entre otras cosas una cuota cada vez más importante de empleos y pseudoempleos "femeninos" y "juveniles", con problemas específicos, que debe tener voz propia en las organizaciones de los trabajadores. No abrirse hacia estas nuevas realidades del mundo del trabajo significa aceptar como un hecho irreversible la escasísima presencia sindical entre las mujeres y entre los trabajadores de las nuevas generaciones.

Por lo que se refiere al tema de la formación, la cuestión es todavía más compleja. En España no se realizan investigaciones sistemáticas sobre la evo-

19. Garonna, P. (ed.), Il margine e la voce. I giovani nel mercato del lavoro e nelle relaxioni industriali dei paesi avanzati, Franco Angeli, Milản, 1988. 
lución de las cualificaciones laborales. No se sabe a ciencia cierta cómo está repercutiendo el cambio tecnológico sobre el contenido de los empleos, sobre la calidad del trabajo. Situación que contrasta, por ejemplo, con los esfuerzos y recursos dedicados a confeccionar el índice de precios al consumo. Las aproximaciones al tema utilizan como punto de referencia las titulaciones educativas de la población ocupada. Esto nos puede dar una idea del nivel de cualificación del individuo empleado, pero sólo una idea muy sesgada acerca del contenido (en cuanto a exigencias de cualificación) del empleo ocupado por ese individuo. Sin embargo, se insiste hasta la saciedad en que muchos jóvenes no alcanzan los niveles de cualificación requeridos por los empleos, a pesar de que el nivel medio de titulaciones educativas de las nuevas generaciones es superior al de los adultos actualmente en activo. Cabe preguntarse a qué se debe esta paradoja y por qué, en cualquier caso, se produce una clamorosa unanimidad - tanto en el dominio político como en el ideológico y científico- a la hora de demandar más formación para los jóvenes, que es valorada así como la panacea del paro juvenil.

Intuyo cuatro tipos de razones. En primer lugar, por parte de muchas unidades productivas, la pretensión de reducir los costes de formación/adiestramiento de los nuevos contratados descargándolos sobre el conjunto de la sociedad, operación para la que se utiliza como excusa el nivel de paro jtwenil. Al parecer, lo que antes de la crisis económica se solía hacer dentro de las unidades productivas, ahora debe hacerse fuera y a cargo de otras instancias sociales. En segundo lugar, el hecho de que la formación es un negocio que mueve miles de millones de peseras en el que participan diferentes instancias estatales con sus honestos y eficaces funcionarios, empresas privadas guiadas por el objetivo de ofrecer el mejor servicio a los alumnos y obtener el máximo beneficio, y las organizaciones sindicales y patronales con sus múltiples, contradictorios $y$ legítimos intereses. En tercer lugar, la creencia ampliamente generalizada de que vamos hacia un tipo de sistema productivo renovado donde tecnologias avanzadas y procesos organizativos cada vez más complejos van a exigir mano de obra con cualificaciones superiores y distintas. Este diagnóstico contrasta con la sospecha fundada de que, hoy por hoy, muchos jóvenes están desempefrando actividades de categoría inferior a su nivel de titulación/cualificación.

Los futuribles sobre la cantidad y calidad de empleos, que requerirá el sistema productivo de pasado mañana, han de apoyarse sobre el análisis riguroso de las tendencias manifestadas en el mercado de trabajo durante el pasado inmediato y actualmente en curso, tal como hace, por ejemplo, el Departamento de Estadísticas Laborales estadounidense ${ }^{20}$. A falta de estos análisis, pueden

20. Levin, H. y Rumberger, R.W. "Requisitos educativos para el futuro mercado de rra" bajo", en $\mathrm{J}$. Grao (ed.), Planificación de la educación y mercado de trabajo, Narcea, Madrid, 
formularse diversas hipótesis de evolución fundamentadas todas ellas en la observación de algunos procesos en curso. Es posible que el nuevo sistema productivo esté caracterizado, efectivamente, por más y mejores empleos, es decir, por un nivel de empleo global superior con una cuota mayor de empleos cualificados y una cuota menor de empleos no cualificados. También es posible que esa redistribución de las cualificaciones se dé con un nivel global de empleo estacionario o en regresión. Pero resulta igualmente razonable conjeturar un mercado de trabajo caracterizado todavía por el paro masivo y por una acentuación de las tendencias a la polarización de las cualificaciones (desaparición de las cualificaciones intermedias, que serían absorbidas en la mayor parte por las inferiores y en la menor por las superiores). En el límite podría hipotizarse incluso la evolución hacia un mercado de trabajo caracterizado por la elevada cualificación de todos sus recursos humanos y una tasa de actividad mínima (por ejemplo en torno al $20-25 \%$ de la población total).

Todos estos escenarios son posibles, tanto los sesgados hacia un futuro optimista como los sesgados hacia un futuro pesimista. En estas circunstancias resulta relevante preguntarse, en primer lugar, si hay algunos procesos básicos que van a producirse de todas formas (o que hay que procurar que se produzcan), independientemente del escenario que llegue a hacerse realidad efectivamente. A mi entender, hay al menos un proceso que debe ser valorado como inevitable: la modernización del tejido productivo. Es legítimo desear que esta vaya acompañada de más y mejores empleos. Pero es razonable temer que pueda desarrollarse en un contexto de paro masivo y degradación de la mayoría de los empleos. Hay que preguntarse también, en segundo lugar, acerca de las implicaciones de la modernización del tejido productivo para la política de formación. La respuesta es clara: un tejido productivo modernizado - condición sine qua non para ocupar un lugar no periférico en la economía mundial - exige una cuota significativa (aunque «indeterminada») de recursos humanos responsables, motivados y cualificados. Por lo tanto, hay que dar más formación, una formación articulada teniendo en cuenta las señales (inevitablemente contradictorias) que lance el sistema productivo. En consecuencia, una formación que debe ocupar una posición más relevante entre las estrategias de política económica.

Ahora bien, en economías intermedias como la española existe una alta probabilidad de que la modernización del tejido productivo llegue acompañada del escenario más sombrío imaginable. En estas circunstancias hay que volver a preguntarse qué hacer con la formación, lo que nos introduce en el

1988; Riche, R.W. y otros, "High Technology Today and Tomorrow: A Small Slice of the Employment Pie», en Monthly Labor Review, noviembre, 1983; Silvestri, G.S. y otros, "Occupational Employment Projections Through 1995", en Monthly Labor Review, noviembre, 1983. 
cuarto tipo de razones que explican la demanda generalizada de más y mejor formación. Incluso aceptando que los escenarios pesimistas son los más problables para mañana y pasado mañana, hay que apostar por una política de mercado de trabajo vertebrada en torno a la idea de dar más y mejor formación al conjunto de la población. Pero ahora, las estrategias de formación estarían orientadas, en primera instancia, por criterios de "política social», y sólo en un segundo nivel por criterios de política economica ${ }^{21}$. Supongamos que, por el procedimiento científico más riguroso a nuestro alcance, conseguimos descubrir que el mercado de trabajo del año 2000 va a necesitar un $25 \%$ de superproductores y un $75 \%$ de auxiliares descualificados, y que la tasa de paro se situará en torno al $10 \%$. Aun en este caso recomiendo un esfuerzo máximo para incrementar el nivel general de formación de la población. Por muchas y variadas razones.

En primer lugar, por las ya apuntadas de política económica en relación con la exigencia de modernización del tejido productivo. En segundo lugar, porque la formación sigue siendo una de las mejores alternativas vitales para quienes no caben en el mercado de trabajo, lo cual es particularmente cierto en el caso de los jóvenes. $Y$ si se racionalizan los aparatos formativos puede ser incluso una alternativa atractiva para la mayoría. En tercer lugar, porque la evolución efectiva de las cualificaciones depende también del nivel de cualificación de la fuerza de trabajo. Un trabajador cualificado tiene más posibilidades de acabar modificando las condiciones de un empleo banal que un trabajador no cualificado. En cuarto lugar, porque el desarrollo de los sistemas, de formación es uno de los más importantes generadores directos de empleo en toda economía moderna (el empleo de los formadores, administradores y subalternos) y uno de los mecanismos más importantes de reducción de la tasa de actividad (por definición, quien está en formación no está en paro). Por último, aunque pueden apuntarse muchas más, porque lo menos que puede hacerse con los probables excluidos del sistema económico modernizado es darles una formación que les permita defenderse de manera socialmente digerible en la jungla hacia la que vamos. Por ejemplo, que les permita organizarse para exigir el acceso a los diferentes servicios públicos con el único requisito de haber nacido, y para conseguir una renta mínima de ciudadanía que los mantenga en el umbral de una digna pobreza. Si además de excluidos han de ser analfabetos funcionales, porque las necesidades del sistema económico no justifican la inversión en su formación, habrá que modificar al alza cualquier previsión que se haga sobre las necesidades futuras de mano de obra en el capítulo de vigilancia y seguridad ciudadanas.

21. Offe, C. Lo stato nel capitalismo maturo, Etas libri, Milán, 1977. 
Así pues, existen muchas e incluso muy buenas razones para estar a favor de dar más formación. En la situación actual y en relación con los jóvenes, esto significa defender la articulación de dispositivos de inserción que definan una franja intermedia entre los inacrivos escolarizados y los activos ocupados en las posiciones más fuertes, de manera que la reducción de la tasa de actividad se vea acompañada de la aparición de canales alternativos de integración social. Esta franja intermedia no puede convertirse en un nuevo almacén de fuerza de trabajo sobrante. Hay que dotarla de contenido para que pueda cumplir dos objetivos. En primer lugar, ocupar a la gente en actividades socialmente útiles, y que además sean formativas, al margen de lo que en Suecia se ha dado en llamar el umercado libre de trabajo». En segundo lugar, permitir a los jóvenes que puedan construirse su identidad social a pesar de no haber podido integrarse en ese mercado libre de trabajo.

Recapitulemos explicitando cuáles son, a mi entender, las consecuencias derivadas de este análisis en cuanto a la situación de los jovenes en un mercado de trabajo como el español y en cuanto a la política de empleo juvenil actualmente vigente. En primer litgar, resulta dificil encontrar razones que justifiquen las medidas encaminadas a reducir cl coste de la fuerza de trabajo juvenil. Desde luego, resulta dificil justificar la actual proliferación de modalidades de contratación no indefinida. Hay más de una docena, lo que nos ha convertido en el pás mejor dotado en este sentido de la OCDE y, simultáneamente, en el que ha alcanzado la tasa de precarización del mercado de trabajo más elevada.

Los dispositivos específicos de inserción sólo se justifican cuando hay de por medio un problema de formación. Este problema puede plantearse a tres niveles: educación básica, cualificaciones profesionales genéricas, y destrezas específicas de una unidad productiva concreta o de un puesro de trabajo específico. En principio, los dos primeros niveles no tienen por qué afectar a las unidades productivas; hacen referencia a problemas que deben ser abordados por otras instancias sociales y cuyo tratamiento implicaría una reducción de la rasa de actividad. Ahora bien, existen razones incluso de orden pedagógico que aconsejan -al menos para algunos grupos de jóvenes- una terapia formativa desarrollada en estrecha conexión con las unidades productivas. En este caso parece lógico aceptar la existencia de algún tipo de compensación económica para las empresas. Por lo que se refiere al tercer nivel, se trata de un tipo de problemas que sólo pueden resolverse mediante un período de rodaje in situ. Dadas las circunstancias actuales, podría aceptarse la conveniencia de mantener salarios diferenciados (directamente o subvencionando una parte) mientras el joven recién contratado alcanza la productividad estándar.

Mi conclusión es, por tanto, que hay que respetar algunos (pocos) dispositivos de inserción encaminados a despejar carencias en formación, primando a las empresas que se acojan a ellos. A condición, claro está, de que las empre- 
sas ofrezcan empleos cualificados o al menos susceptibles de involucrar a los jóvenes en un irinerario laboral con perspectivas. La función de estos dispositivos sería la de trazar un camino crítico que permita a una cuota relevante de jóvenes acceder al mercado de trabajo evitándoles caer en uno de estos dos extremos, a saber, la exclusión absoluta del mercado de trabajo o la superexplotación en ocupaciones degradadas, sin futuro y ni tan siquiera remuneradas con un salario "digno". Lo cual implica que el uso de los dispositivos de inserción debe estar sometido a un control riguroso por parte de la inspección laboral o, en su defecto, de las instancias sindicales. Si en nuestro sistema de relaciones industriales hay alguna práctica irregular necesitada de urgente erradicación es, antes que cualquier otra, la de beneficiarse de sustanciosas bonificaciones por contratar a jóvenes en "formación" para luego tenerlos ocupados en actividades absolutamente banales, despedirlos antes de que se materialice la obligación de estabilizarlos y volver a iniciar el proceso con la generación siguiente. 